TRANSVERSE SPACE-CHARGE EFFECTS

in the AGS BOOSTER

DURING INJECTION

$A D$

Booster Technical Note

No. 120

April 29, 1988

E.P. Colton, D. Shi (LANL)

\&

Z. Parsa

ACCELERATOR DEVELOPMENT DEPARTMENT

Brookhaven National Laboratory

Upton, N.Y. 11973 


\title{
TRANSVERSE SPACE-CHARGE EFFECTS IN THE AGS BOOSTER DURING INJECTION
}

\author{
E. P. Colton, D. Shi, Los Alamos National Lab
}

Z. Parsa, Brookhaven National Laboratory

\begin{abstract}
We have analyzed the transverse motion for $200 \mathrm{MeV}$ protons under strong space-charge conditions. We considered up to $5 \times 10^{12}$ protons per bunch; for the assumed distribution this corresponds to a maximum tune shift of -0.75. We utilized single particle tracking to study the motion in normalized phase space as a function of initial particle amplitude. Subsequent FFT analyses were used to obtain the fractional betatron tunes at different $\mathrm{z}$ values along the bunch. The motion shows $x-y$ coupling due to the so-called Montague resonance $\left(2 Q_{x}-2 Q_{y}\right.$ $=0$ ). Perturbations arise when particles have tunes in the neighborhood of the $2 Q$ $=9$ half-integral resonances but losses do not occur. It appears that the motion is stabilized simply due to the strong amplitude dependence of the tunes.
\end{abstract}

\section{Introduction}

The AGS booster ${ }^{1}$ will be a multipurpose facility with the option to accelerate protons as well as heavy ions. The ultimate goal for protons will be to accelerate $3 \cdot 10^{13}$ protons per pulse (ppp) from 200 to $1500 \mathrm{MeV}$ kinetic energy at a repetition rate of 7.5 $\mathrm{Hz}$. This corresponds to a time averaged current of 36 microamperes. Attainment of such a goal requires stable motion of the beam. In this note we study the amplitude dependence of the betatron tumes for single particles. There are a number of questions 
that we intend to pursue with this analysis: (a) What is the maximum tune depression that takes place for a given bunch population? (b) How is the beam populated with respect to tune at different positions along the bunch? (c) What happens in the neighborhood of betatron resonances? (d) In regard to (c) are there measureable losses?

The analyses undertaken were for protons in the AGS booster circulating at a kinetic energy of $200 \mathrm{MeV}$. The motion was studied using a locally modified version of the program FRANKENSPOT. ${ }^{(2)}$ The bunch is taken to be a three dimensional ellipsoid populated according to a tri-gaussian distribution; the charge density being given by

$$
\rho(x, y, z)=\frac{1}{(2 \pi)^{3 / 2} \sigma_{x} \sigma_{y} \sigma_{z}} \exp \left[-\frac{1}{2}\left\{\left(\frac{x}{\sigma_{x}}\right)^{2}+\left(\frac{y}{\sigma_{y}}\right)^{2}+\left(\frac{z}{\sigma_{z}}\right)^{2}\right\}\right]
$$

We have implemented a fast Fourier transform (FFT) into the program so as to be able to view the motion in the frequency domain. We have modified the spacecharge calculation so as to include the effects of image forces (which are minor in this application). Additionally the dipole routines now allow for non-normal entry and exit with appropriate thin-lens edge-focussing terms included.

The parameters of the AGS booster are listed in Table 1 for the initial analysis.

It is assumed that the harmonic $3 \mathrm{rf}$ system has a net voltage of $90 \mathrm{kV}$ and that the beam is not being accelerated. The studies reported here do not include synchrotron oscillations. We study the motion of monoenergetic particles. The values of $\beta$ and $\alpha$ listed in Table 1 are expressed at the observation point in the ring. Initially we considered just particles at the longitudinal center of the bunch $(z=0)$. Later in the work we explore the tune dependence of particles located elsewhere along the bunch. These studies are explained below.

\section{Tracking Studies}

This part of the analysis used the parameters listed in Table 1, and considered particles located at the center of the bunch $(z=0)$. The particles were tracked using FRANKENSPOT. ${ }^{2}$ The initial coordinates of the particles always had $\mathrm{x}=\mathrm{y}$ and 
Table 1: AGS BOOSTER PARAMETERS

Circumference
Proton kinetic energy
Betatron tunes $\left(\mathrm{Q}_{x}, \mathrm{Q}_{y}\right)$
Number of bunches
Protons per bunch
rms momentum spread $\left(\sigma_{p} / p\right)$
rms bunch length $\left(\sigma_{l}\right)$
rms normalized emittance
$\beta_{x}, \beta_{y}$
$\alpha_{x}, \alpha_{y}$

$201.78 \mathrm{~m}$

$200 \mathrm{MeV}$

$4.82,4.83$

3

$5 \times 10^{12}$

$0.15 \%$

10. $\mathrm{m}$

$8.6 \mathrm{~mm}-\mathrm{mrad}$

$7.27 \mathrm{~m}, 7.29 \mathrm{~m}$

$-1.30,1.26$

$x^{\prime}=y^{\prime}=0$ and $d p / p=0$. Figures $1(a)$ and $1(b)$ show normalized phase space plots of the motion for the example of three particles with initial coordinates $x=y=4 \mathrm{~mm}$, $\mathrm{x}=\mathrm{y}=6 \mathrm{~mm}$, and $\mathrm{x}=\mathrm{y}=8 \mathrm{~mm}$. In Fig. 1(a) the coordinates $\mathrm{x}_{1}=\mathrm{x}$, and $\mathrm{x}_{2}=\beta_{x}$ $\mathrm{x}^{\prime}+\alpha_{x} \mathrm{x}$; correspondingly $\mathrm{y}_{1}=\mathrm{y}$ and $\mathrm{y}_{2}=\beta_{y} \mathrm{y}^{\prime}+\alpha_{y} \mathrm{y}$ in Fig. $1(\mathrm{~b})$. The $\beta$ and $\alpha$ values are given in Table 1 . The structure in Fig. 1 is rich - the particles started at $8 \mathrm{~mm}$ occupy a large band. In this case the depressed tune is near 4.5 , i.e. in the neighborhood of the $2 \mathrm{Q}=9$ betatron resonances. The particles are not lost over the 200 turns of tracking.

The FFT analyses were performed on the $x(t)$ and $y(t)$ values for 200 points; the argument $t$ represents time. Figure 2 shows the results for the particles started with $x=y=4 \mathrm{~mm}$ and $x=y=8 \mathrm{~mm}$. The horizontal scales represent the fractional tunes (df); the inferred tunes are $4+\mathrm{df}$ or 5 - df. The vertical scale is the logarithm of the FFT signal. The FFTs shown in Figs. 2(a) and 2(b) each show the main depressed tunes at $\mathrm{Q}=4.21$ as well as secondary peaks at $\mathrm{df}=0.28$; the motion for the $\mathrm{x}=\mathrm{y}=4 \mathrm{~mm}$ particle can be described with just two frequencies. The FFT results for the $x=y=8$ $\mathrm{mm}$ particle are shown in Figs. 2(c) and 2(d); the peak of the distribution is near $\mathrm{df}=0.45$ but the spectrum is quite wide indicating sampling over a wide range of amplitudes as is evident in Fig. 1. Similar analyses were performed for a wide range of initial $\mathrm{x}=\mathrm{y}$ values from $0.5 \mathrm{~mm}$ to $35 \mathrm{~mm}$. The results of the FFT runs are given in Table 2. 
Table 2: FFT RESULTS FOR THE INDICATED STARTING $\mathrm{X}=\mathrm{Y}$ VALUES

\begin{tabular}{c|c|c|c|c}
$\mathrm{x}=\mathrm{y}$ & $\mathrm{df}(\mathrm{x})$ & $\mathrm{Q}_{x}$ & $\mathrm{df}(\mathrm{y})$ & $\mathrm{Q}_{y}$ \\
\hline$(\mathrm{mm})$ & & & & \\
0.5 & 0.135 & 4.135 & 0.110 & 4.110 \\
1.0 & 0.140 & 4.140 & 0.115 & 4.115 \\
2.0 & 0.162 & 4.162 & 0.135 & 4.135 \\
4.0 & 0.211 & 4.211 & 0.210 & 4.210 \\
6.0 & 0.300 & 4.300 & 0.302 & 4.302 \\
8.0 & 0.45 & 4.45 & 0.45 & 4.45 \\
10.0 & 0.48 & 4.52 & 0.48 & 4.52 \\
12.0 & 0.445 & 4.555 & 0.445 & 4.555 \\
15.0 & 0.370 & 4.630 & 0.370 & 4.630 \\
18.0 & 0.319 & 4.681 & 0.319 & 4.681 \\
20.0 & 0.295 & 4.705 & 0.296 & 4.704 \\
25.0 & 0.255 & 4.745 & 0.255 & 4.745 \\
30.0 & 0.232 & 4.768 & 0.230 & 4.770 \\
35.0 & 0.217 & 4.783 & 0.220 & 4.780
\end{tabular}

The data listed in Table 2 are also plotted in Fig. 3(a) where we plot $Q_{x}$ and $\mathrm{Q}_{y}$ vs starting amplitude $\mathrm{x}=\mathrm{y}$. There are uncertainties of about \pm 0.07 in $\mathrm{Q}_{x}$ and $\mathrm{Q}_{y}$ at $\mathrm{x}=\mathrm{y}=8 \mathrm{~mm}$ and $10 \mathrm{~mm}$ (see Fig. 2). The results shown in Fig. 3(a) can be weighted by the assumed tri-gaussian distribution [Eq. (1)] and we show in Fig. 3(b) the distribution of population vs tune at $\mathrm{z}=0$. The weight is simply $\exp \left(-\left(\mathrm{x} / \sigma_{\mathfrak{x}}\right)^{2}\right)$ where we have estimated $\sigma_{x}$ to be $9.5 \mathrm{~mm}$ at the observation point; this is based upon the rms normalized emittance of $8.6 \mathrm{~mm}$-mrad. Figure $3(\mathrm{~b})$ shows that the data heavily populate tunes near the minimum depressed value. This observation is generally true independent of the actual charge distribution (as long as there are central concentrations). The actual tune population of the beam for the entire bunch will not be as steep as shown in Fig. 3(b) if we include contributions from other locations along the bunch. 


\section{Table 3: FFT RESULTS FOR THE Z POSITIONS SHOWN}

Flux $2.5 \times 10^{12}$ protons per bunch and $\sigma_{z}=13.5 \mathrm{~m}$.

Depressed $Q$ value for given $z$.

\begin{tabular}{c|c|c|c}
$\begin{array}{c}\mathrm{x}=\mathrm{y} \\
(\mathrm{mm})\end{array}$ & $\mathrm{z}=0 \mathrm{~m}$ & $\mathrm{z}=13.5 \mathrm{~m}$ & $\mathrm{z}=27.0 \mathrm{~m}$ \\
\hline 1.47 & 4.575 & 4.676 & 4.782 \\
2.94 & 4.586 & 4.681 & 4.785 \\
4.40 & 4.599 & 4.689 & 4.787 \\
5.87 & 4.625 & 4.700 & 4.790 \\
8.81 & 4.675 & 4.727 & 4.795 \\
11.74 & 4.716 & 4.758 & 4.802 \\
17.61 & 4.765 & 4.782 & 4.811
\end{tabular}

\section{Lower Intensity Studies}

We carried out further analyses of the motion in order to compare results from different $z$ positions along the bunch. We reduced the intensity to $2.5 \times 10^{12}$ protons per bunch and increased the rms bunch length to $13.5 \mathrm{~m}$ so as to keep the depressed tunes above 4.5 (thereby eliminating smearing due to $2 Q=9$ resonances). Seven particles were started and tracked, as above, with $\mathrm{x}=\mathrm{y}$ at each of three locations $\mathrm{z}=0,13.5 \mathrm{~m}(1 \sigma)$, and $27.0 \mathrm{~m}(2 \sigma)$. FFT analyses were carried out upon the $\mathrm{x}(\mathrm{t})$ and $\mathrm{y}(\mathrm{t})$ data. The depressed tunes for $Q_{x}$ and $Q_{y}$ were essentially identical in each case so we just present the value for $Q_{x}$ which we call $Q$. The results are given in Table 3 and in Fig. 4(a). We weight the results by the particle distribution function

$$
\exp \left[-\left(\left(\frac{x}{\sigma_{x}}\right)^{2}+\frac{1}{2}\left(\frac{z}{\sigma_{z}}\right)^{2}\right)\right]
$$

and the results are plotted in Fig. 4(b). The relative population vs tunes appear to line up.

Next we fixed $\mathrm{x}=\mathrm{y}=1.5 \mathrm{~mm}$ and redid the studies for different $\mathrm{z}$ values for $0<\mathrm{z}<2.5 \sigma_{z}$ 
Table 4: FFT RESULTS FOR $\mathrm{X}=\mathrm{Y}=1.5 \mathrm{MM}$

\begin{tabular}{c|c|c}
$\begin{array}{c}\text { z position along } \\
\text { the bunch }(\mathrm{m})\end{array}$ & $\mathrm{Q}$ & relative population \\
\hline 0 & 4.573 & 0.984 \\
3.375 & 4.579 & 0.954 \\
6.750 & 4.597 & 0.868 \\
10.125 & 4.636 & 0.743 \\
13.500 & 4.672 & 0.597 \\
16.875 & 4.704 & 0.451 \\
20.250 & 4.736 & 0.319 \\
23.625 & 4.767 & 0.213 \\
27.00 & 4.782 & 0.133 \\
33.75 & 4.805 & 0.043
\end{tabular}

in order to see how the $Q$ values vary longitudinally down the bunch centerline. As before the FFT analyses were performed upon the transverse coordinates and the depressed $Q$ values were obtained. Table 4 lists the results and the data are plotted in Fig. 5(a). The $Q$ values are weighted by the function

$$
\exp \left[-\left(.0161+\frac{1}{2}\left(\frac{z}{\sigma_{z}}\right)^{2}\right)\right]
$$

and we plot the relative populations vs tune in Fig. 5(b).

\section{Discussion}

The assumption of a trigaussian distribution with $\sigma_{z}=10.0 \mathrm{~m}$ results in maximum transverse tune shifts of approximately -0.75 for $5 \times 10^{12}$ protons per bunch at $200 \mathrm{MeV}$ kinetic energy. The attainment of higher intensities will require flatter distributions and somewhat longer bunches. It is improbable that we can cross the integer $Q=4$ resonances without beam losses. Whatever the maximum tune shifts are we must bear in mind that the beam populations are enhanced down at the minimum depressed tunes. These regions should be free of strong betatron resonances. 
A fraction of the beam will cross the half-integral resonances $2 Q=9$ with some emittance growth; it is recommended that quadrupole errors be kept to a minimum. With regard to third-integer resonances it follows that the sextupolar errors present in dipole fields, and induced by eddy curents in vacuum chambers, be kept in check.

We recommend that further tracking studies of this machine incorporate FFT algorithms for the purpose of understanding single particle dynamics in the presence of nonlinear forces.

\section{Acknowledgement}

One of us (Z. P.) would like to thank Los Alamos National Laboratory for its hospitality during summer 1987 when most of this work was carried out.

\section{Reference}

1. See, e.g., Z. Parsa, BNL-39311 (1987); AGS Booster Design Manual.

2. See e.g., Miguel A. Furman, "Effect of the Space-Charge Force on Tracking at Low Energy," Report SSC-115 (198T).

\section{$7 \quad$ Figure Captions}

Figure 1. Normalized phase-space distributions of the coordinates of three particles tracked for 200 turns. The initial coordinates of the particles were $x=y=4 \mathrm{~mm}, 6 \mathrm{mmn}$. and $8 \mathrm{~mm}$. The $x^{\prime}=y^{\prime}=0$ in each case. The conditions of the machine are given in Table 1.

Figure 2. FFT results from the data shown in Fig. 1 for the $x=y=4$ mm and $\&$ mm cases.

Figure 3. (a) Depressed tunes $Q_{x}$ and $Q_{y}$ vs initial starting amplitude for particles at the bunch longitudinal center. (b) Relative population vs tune at $z=0$. 
Figure 4. (a) Depressed tunes $\mathrm{Q}$ vs initial starting amplitude at the three $\mathrm{z}$ positions along the bunch. Proton flux $2.5 \times 10^{12}$ per bunch and rms bunch length $\sigma_{z}=13.5 \mathrm{~m}$. (b) Corresponding relative populations vs tune at the three $z$ positions.

Figure 5. (a) Depressed tunes $\mathrm{Q}$ vs $\mathrm{z}$ position along the bunch for fixed $\mathrm{x}=\mathrm{y}=1.5$ $\mathrm{mm}$. Proton flux $2.5 \times 10^{12}$ per bunch and $\sigma_{z}=13.5 \mathrm{~m}$. (b) Corresponding relative population vs tune (along the bunch). 

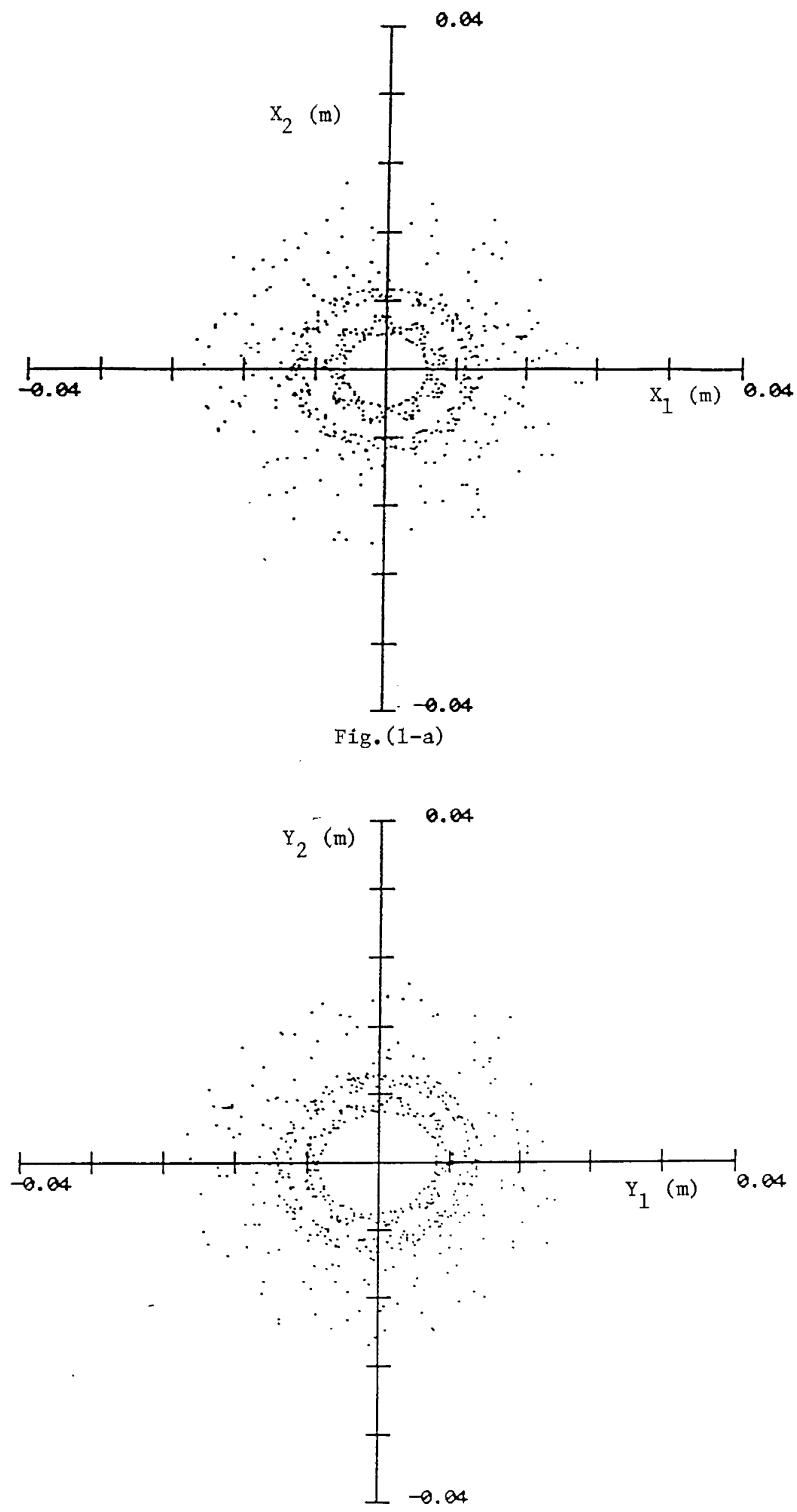

Fig. (1-b) 

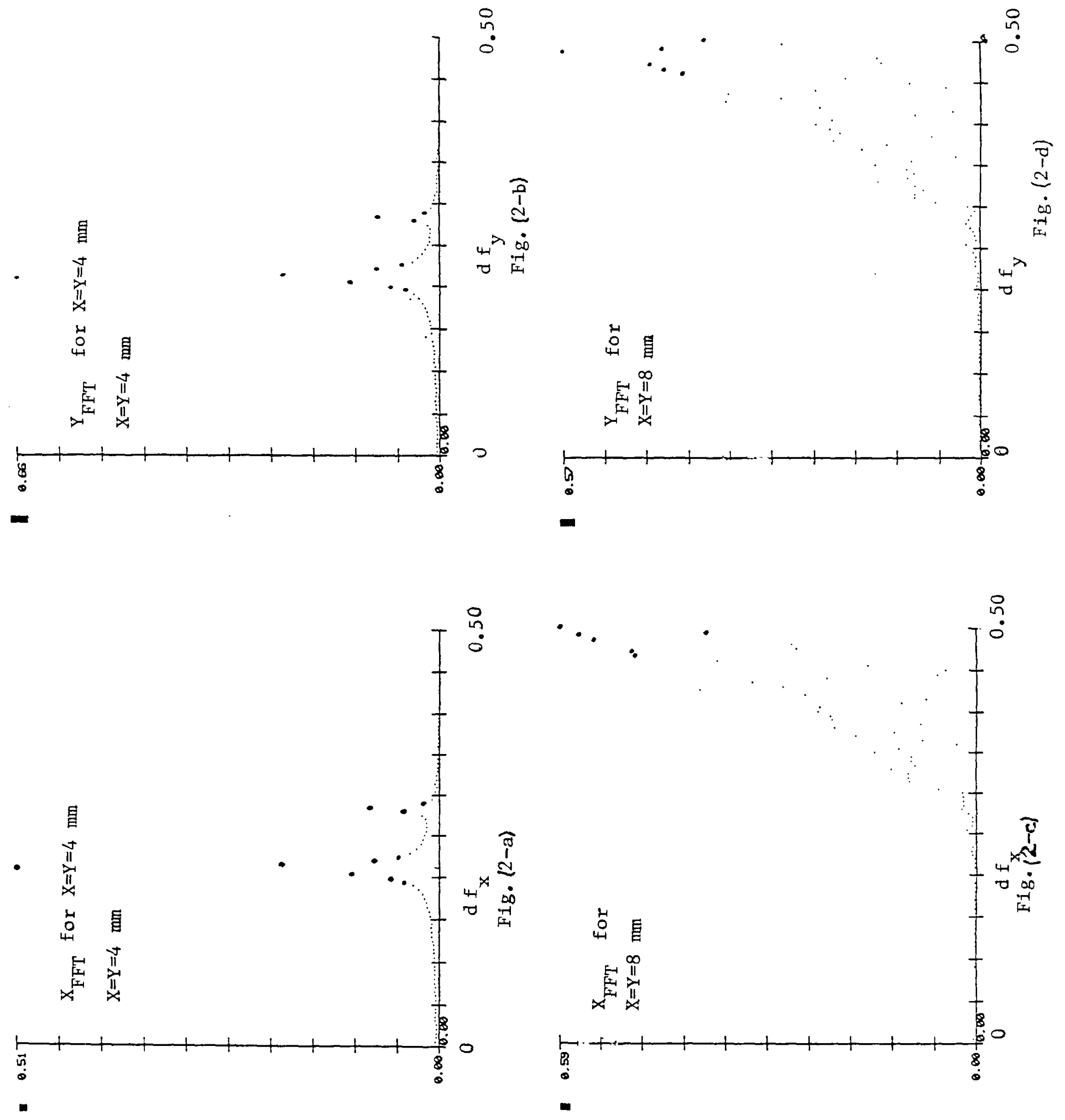

Fig. 2 

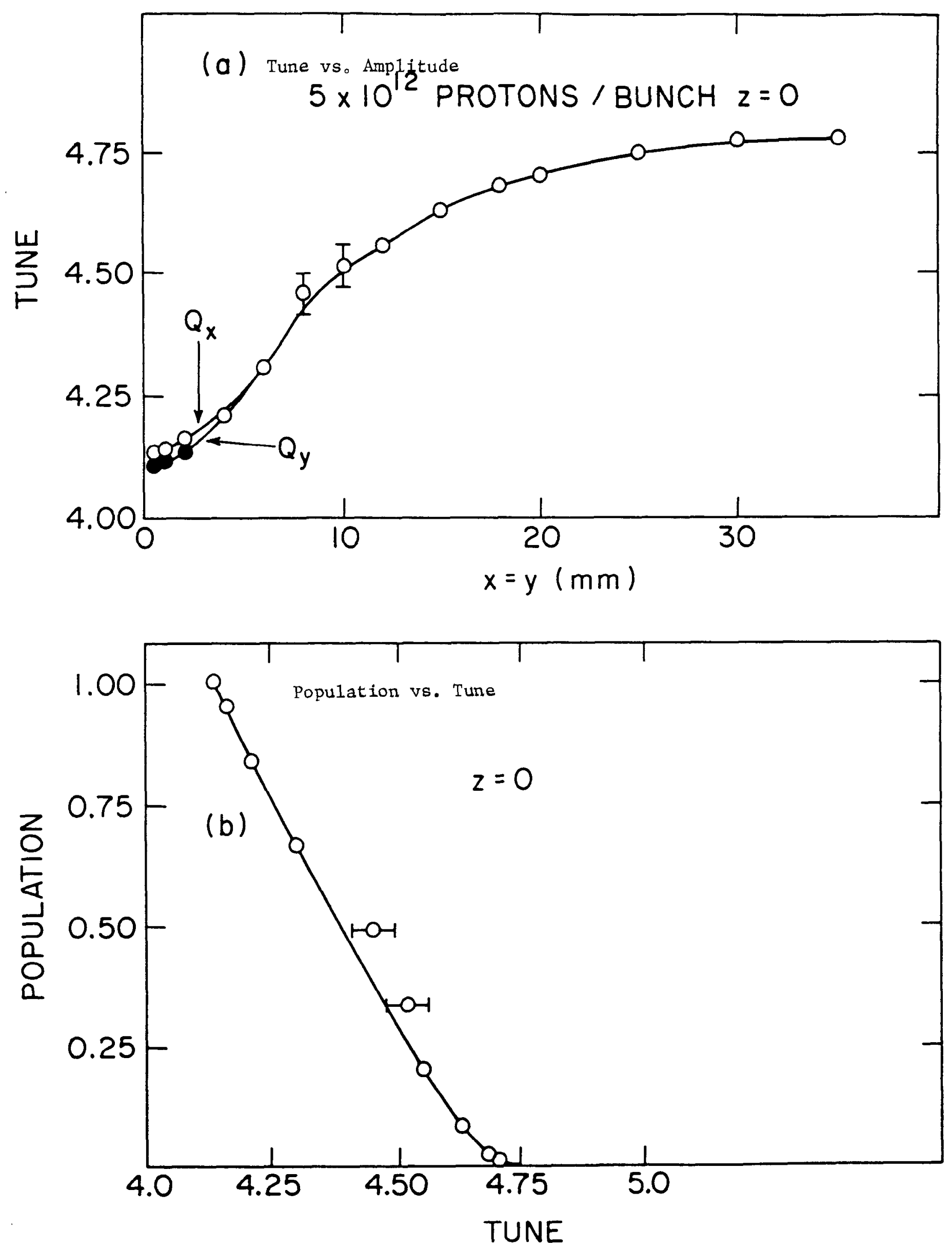

Fig. 3 

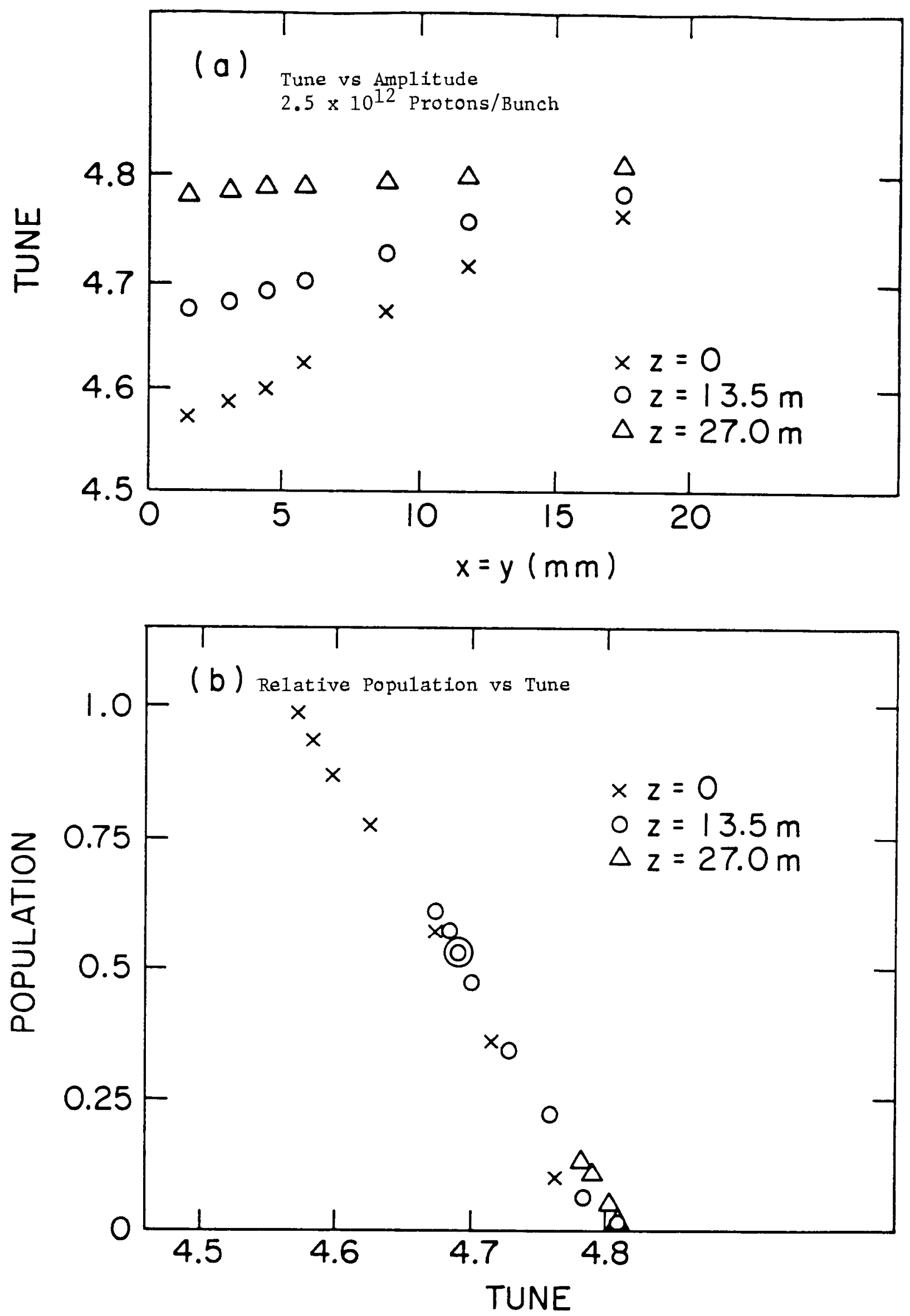

Fig. 4 

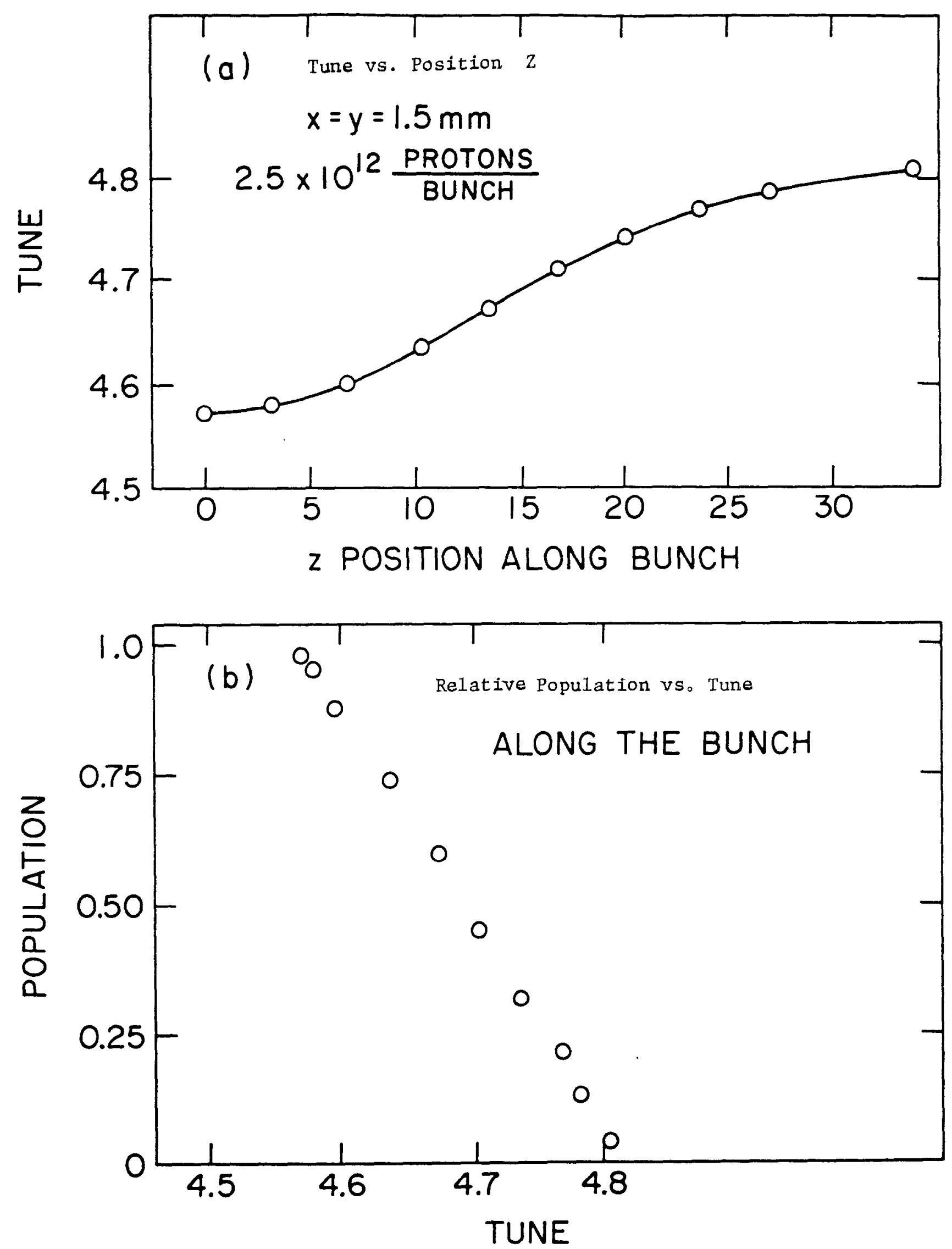

Fig. 5 